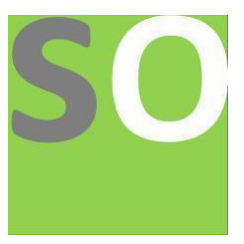

Article title: The Buoyant Brain

Authors: Mohamed Abdelrahman Abdalla[1]

Affiliations: Neurosurgery Department, St George's university hospital, Blackshaw road, London, UK[1]

Orcid ids: 0000-0002-1889-5055[1]

Contact e-mail: maelsadek@yahoo.com

License information: This work has been published open access under Creative Commons Attribution License $\mathrm{http}: / / c r e a t i v e c o m m o n s . o r g / l i c e n s e s / b y / 4.0 /$, which permits unrestricted use, distribution, and reproduction in any medium, provided the original work is properly cited. Conditions, terms of use and publishing policy can be found at https://www.scienceopen.com/.

Preprint statement: This article is a preprint and has not been peer-reviewed, under consideration and submitted to ScienceOpen Preprints for open peer review.

DOI: 10.14293/S2199-1006.1.SOR-.PPWDRBO.v1

Preprint first posted online: 29 January 2021

Keywords: Buoyancy, CSF functions, Ventricular functions, communicating hydrocephalus 


\title{
The buoyant brain
}

\begin{abstract}
:
CSF is believed to provide a buoyancy medium to protect the brain against trauma and to reduce its weight ${ }^{1}$. However, the origin of this belief, how this buoyancy works and its implications in various CSF disorders have not got enough attention in the literature.

This study aimed to review the literature for the previous works that investigated the buoyancy environment of the brain. By applying the laws of buoyancy to the intracranial system, possible explanations of the common CSF disorders were suggested and compared to the current knowledge.
\end{abstract}

\section{Key words:}

Buoyancy, CSF functions, ventricular functions. Communicating hydrocephalus

\section{Introduction:}

Our understanding of CSF formation, flow and drainage has evolved through the last century. The concept of unidirectionally circulating CSF from sources to drainage sites has fallen out of favour due to the new accumulating findings ${ }^{1}$. Closely related are the ventricles and their undefined functions in the adult life. Nonetheless, the laws of buoyancy physics seem to play a crucial role in how this system could be operating.

\section{Does the brain float in CSF?}

Buoyancy provided by CSF is listed in many papers as one of the CSF functions but with no clear reference to the origin of this doctrine. This buoyancy is believed to cause $97 \%$ reduction of the weight of the brain ${ }^{1}$ to become $25-50$ grams $^{3}$ or less specifically less than 100 grams $^{4}$. As it is practically impossible to weigh the living brain without disrupting its buoyancy environment, these assumptions are probably based on pure calculations. I have not been able to find any study which weighed the brain in a CSF-like medium in vitro to prove these numbers.

Mathematically, however, this can be proven if the densities of both CSF and brain are known. There have been a few studies that tried to identify the density of the brain and its components ${ }^{5}$. For simplicity, the human cerebrum density is estimated to be 1.0417 gram per cubic centimetres for males and 1.0411 gram per cubic centimetres for females ${ }^{6}$. The specific gravity of CSF on the other hand is 1.004 to $1.012^{7}$. From these numbers, CSF buoyancy can neutralize $97 \%$ of the brain weight which equates roughly to 50-100 grams (the average weight is 1336 grams in males and 1198 grams in females $^{8}$ ) (CSF to brain density is roughly $97 \%$ !).

Aside from the pure academia, buoyancy has got a reasonable clinical attention when mathematical models were developed to compensate for the intraoperative brain shifts. Taking in consideration the loss of buoyant forces $(\mathrm{Fb})$ due to CSF drainage in addition to 
various other factors, these models could update navigation systems with a fair degree of accuracy ${ }^{9,10}$.

Perhaps an easier and more straightforward evidence in this context is our routine neurosurgical experience that the brain resists traction (being pushed) until CSF is drained to then fall easily with gravity. This resisting force is nothing but the underlying Fb.

The concept of the buoyant brain is then taken no further in the literature as far as I am aware.

\section{Functions of the ventricles:}

The ventricles, on the other hand, have a clear embryological function important in neural proliferation and brain development ${ }^{11}$. However, the role of this potentially life-threatening structure in the adult brain is often mixed up with the functions of CSF. Similarly, CSF flow within the ventricles and the periventricular neurons are increasingly recognized to play important roles in the adult life ${ }^{11}$. Nonetheless, all of these functions could have been accomplished without the existence of these expansile organs within the brain. The functions of the ventricles in any animal system are thus not fully recognized ${ }^{11}$.

From the buoyancy perspective, the ventricles are important to buffer any change in either brain or CSF density. For examples, the amount of the blood within the brain increases during systole or expiration ${ }^{11,12}$ which increases its overall density. This means a negative buoyancy state and a caudal movement of the brain which has been shown in different MRI studies ${ }^{13,14,15}$. Firstly, for the increased brain weight secondary to its increased blood volume, more CSF has to be displaced extracranially according to the basic rules of buoyancy physics. This has been shown to be equivalent to half of the added blood volume ${ }^{1}$. Secondly, CSF egresses from the ventricles to further buffer the increased density of the brain. The opposite scenario occurs during diastole and inspiration ${ }^{2,16,17}$ i.e., positive buoyancy.

While the brain pulsatility or the transmission of the capillary pulsations may play a role in these CSF movements ${ }^{13}$, it has been also shown that respiration has a more profound effect on such movements ${ }^{17}$. Nonetheless, the main cause of these observed CSF oscillation seems to be the $\mathrm{Fb}$ which value varies according to the ratio of brain to CSF density. This ratio changes throughout the cardiac or respiratory cycle and in different head positions in addition to various pathological conditions including traumatic brain injury.

A good analogy to this function in the biological systems is the swim bladder in teleost fishes. This structure inflates to decrease the body density as the fish moves to less salinated water to keep itself hovering in the same horizontal plane ${ }^{18}$. Dilated ventricles, on the other hand, cannot cause volume expansion of the whole brain and so still contribute to its increased density. This can be seen in the brain compression associated with ventricular dilatation in communicating hydrocephalus ${ }^{1}$.

Following on with the buoyancy in biological systems, not all fishes, however, have the privilege of this energetically expensive swimbladder. Acanthonus armatus, for example, is a fish which relies on its light body design to stay afloat. Its relatively large head is filled with clear fluid which gives the fish 13-27\% greater lift than if the head had been filled with plasma instead ${ }^{19}$. 


\section{Pathophysiology of the brain buoyancy:}

\section{Subarachnoid haemorrhage:}

This estimated loss of buoyancy in the previous example can help explain the pathophysiology of communicating hydrocephalus after subarachnoid haemorrhage. The increased density of CSF, after mixing with blood, means that a smaller volume of the denser fluid will need to egress from the cranium for the same weight of the brain according to Archimedes. How can this affect the dynamics of CSF absorption or if another pathophysiological element is also implicated is beyond this discussion.

$\mathrm{Fb}$ will increase subsequent to increased CSF density. This creates more resistance to CSF outflow from the ventricles and possibly reflux to cause hydrocephalus (figure 2). The dilated (heavier) ventricles then allow the brain to maintain its buoyancy state in the face of increasing $\mathrm{Fb}$. This is paradoxical to Monro-Killie hypothesis as the subarachnoid haemorrhage is not compensated by CSF escape extracranially but the exact opposite occurs. Moreover, the balance between the brain weight and the $\mathrm{Fb}$ appears to be crucial to the net intracranial pressure as not all of these hydrocephalic cases are associated with increased ICP. Obviously, other types of hydrocephalus can happen after SAH and some of them may cause serious increase in the ICP, but the discussion here is about $\mathrm{Fb}$ and its implication in the low and normal pressure post-SAH communicating hydrocephalic states.

\section{Normal pressure hydrocephalus:}

The above scenario is described as secondary normal pressure hydrocephalus (NPH), so is the communicating hydrocephalus from other causes ${ }^{20}$. These pathophysiological events can be described as positive buoyancy states.

Alike is the "high-riding" looking brain in the shunt responders in idiopathic NPH. More CSF in the Sylvian fissures associated with tight high convexity have been noted to correlate with good outcomes after shunting ${ }^{20,21}$. The pathophysiological events may arise from a relative increased CSF to brain density. Either reduced aging brain weight ${ }^{8}$ or increased protein contents of CSF can result in a positive buoyancy state and subsequently ventriculomegaly. In other words, the brain looks as if it is being pushed upwards or unfolding its telencephalon part.

The weight of dilated ventricles could be notorious to the delicate periventricular vessels. A vicious circle of ischemia and neural loss ${ }^{1}$ resulting in decreased brain density and positive buoyancy and subsequent ventricular dilatation may continue. This is obviously the disease of the physiology which may not be reversed if the treatment is not provided early enough in the course of the problem.

\section{Conclusion:}

Buoyancy plays a role in the brain (and possibly the spinal cord) homeostasis. A balanced brain movement across a horizontal axis is guaranteed by the presence of the ventricular buffer. How this "horizontal balance" is important to the homeostasis of the brain is unclear, 
but more buoyancy targeted treatment could provide better outcomes in various positive and negative buoyancy states than pressure-adjusting only strategies.

\section{References:}

1. Matsumae M, Kuroda K, Yatsushiro S, Hirayama A, Hayashi N, Takizawa K, Atsumi $\mathrm{H}$, Sorimachi T. Changing the Currently Held Concept of Cerebrospinal Fluid Dynamics Based on Shared Findings of Cerebrospinal Fluid Motion in the Cranial Cavity Using Various Types of Magnetic Resonance Imaging Techniques. Neurol Med Chir (Tokyo). 2019 Apr 15;59(4):133-146. doi: 10.2176/nmc.ra.2018-0272. Epub 2019 Feb 28. PMID: 30814424; PMCID: PMC6465527.

2. Stivaros SM, Jackson A. Changing concepts of cerebrospinal fluid hydrodynamics: role of phase-contrast magnetic resonance imaging and implications for cerebral microvascular disease. Neurotherapeutics. 2007 Jul;4(3):511-22. doi: 10.1016/j.nurt.2007.04.007. PMID: 17599715; PMCID: PMC7479718.

3. Adigun OO, Al-Dhahir MA. Anatomy, Head and Neck, Cerebrospinal Fluid. [Updated 2020 Jul 27]. In: StatPearls [Internet]. Treasure Island (FL): StatPearls Publishing; 2020 Jan-. Available from: https://www.ncbi.nlm.nih.gov/books/NBK459286/

4. Bothwell SW, Janigro D, Patabendige A. Cerebrospinal fluid dynamics and intracranial pressure elevation in neurological diseases. Fluids Barriers CNS. 2019 Apr 10;16(1):9. doi: 10.1186/s12987-019-0129-6. PMID: 30967147; PMCID: PMC6456952.

5. Gompertz, R. H. C., (1902), Specific gravity of the brain. The Journal of Physiology, 27 doi: 10.1113/jphysiol.1902.sp000884.

6. Barber TW, Brockway JA, Higgins LS. The density of tissues in and about the head. Acta Neurol Scand. 1970;46(1):85-92. doi: 10.1111/j.1600-0404.1970.tb05606.x. PMID: 4983875.

7. Etherington-Wilson W. Specific Gravity of C.S.F. in Spinal Anaesthesia. Br Med J. 1943;2(4309):165-167. doi:10.1136/bmj.2.4309.165

8. Hartmann P, Ramseier A, Gudat F, Mihatsch MJ, Polasek W. Das Normgewicht des Gehirns beim Erwachsenen in Abhängigkeit von Alter, Geschlecht, Körpergrösse und Gewicht [Normal weight of the brain in adults in relation to age, sex, body height and weight]. Pathologe. 1994 Jun;15(3):165-70. German. doi: 10.1007/s002920050040. PMID: 8072950.

9. M. I. Miga et al., "Model-updated image guidance: initial clinical experiences with gravity-induced brain deformation," in IEEE Transactions on Medical Imaging, vol. 18, no. 10, pp. 866-874, Oct. 1999, doi: 10.1109/42.811265.

10. K. Sun, T. S. Pheiffer, A. L. Simpson, J. A. Weis, R. C. Thompson and M. I. Miga, "Near Real-Time Computer Assisted Surgery for Brain Shift Correction Using Biomechanical Models," in IEEE Journal of Translational Engineering in Health and Medicine, vol. 2, pp. 1-13, 2014, Art no. 2500113, doi: 10.1109/JTEHM.2014.2327628.

11. Lowery, L.A. and Sive, H. (2009), Totally tubular: the mystery behind function and origin of the brain ventricular system. Bioessays, 31: 446-458.

https://doi.org/10.1002/bies.200800207 
12. Dreha-Kulaczewski S, Joseph AA, Merboldt KD, Ludwig HC, Gärtner J, Frahm J. Identification of the Upward Movement of Human CSF In Vivo and its Relation to the Brain Venous System. J Neurosci. 2017 Mar 1;37(9):2395-2402. doi: 10.1523/JNEUROSCI.2754-16.2017. Epub 2017 Jan 30. PMID: 28137972; PMCID: PMC6596847.

13. Greitz D, Wirestam R, Franck A, et al. Pulsatile brain movement and associated hydrodynamics studied by magnetic resonance phase imaging. The Monro-Kellie doctrine revisited. Neuroradiology. 1992 ;34(5):370-380. DOI: 10.1007/bf00596493.

14. Feinberg DA, Mark AS. Human brain motion and cerebrospinal fluid circulation demonstrated with MR velocity imaging. Radiology. 1987 Jun;163(3):793-9. doi: 10.1148/radiology.163.3.3575734. PMID: 3575734.

15. Poncelet BP, Wedeen VJ, Weisskoff RM, Cohen MS. Brain parenchyma motion: measurement with cine echo-planar MR imaging. Radiology. 1992 Dec;185(3):64551. doi: 10.1148/radiology.185.3.1438740. PMID: 1438740.

16. Quencer RM, Post MJ, Hinks RS. Cine MR in the evaluation of normal and abnormal CSF flow: intracranial and intraspinal studies. Neuroradiology. 1990;32(5):371-91. doi: 10.1007/BF00588471. PMID: 2259432.

17. Yamada S, Miyazaki M, Yamashita Y, et al. Influence of respiration on cerebrospinal fluid movement using magnetic resonance spin labeling. Fluids Barriers CNS. 2013;10(1):36. Published 2013 Dec 27. doi:10.1186/2045-8118-10-36

18. Stewart J, Hughes JM. Swim bladder function and buoyancy control in pink snapper (Pagrus auratus) and mulloway (Argyrosomus japonicus). Fish Physiol Biochem. 2014 Apr;40(2):335-46. doi: 10.1007/s10695-013-9846-y. Epub 2013 Aug 25. PMID: 23979722.

19. Horn, M.H., Grimes, P.W., Phleger, C.F. et al. Buoyancy function of the enlarged fluid-filled cranium in the deep-sea ophidiid fish Acanthonus armatus . Mar. Biol. 46, 335-339 (1978). https://doi.org/10.1007/BF00391405

20. Hashimoto M, Ishikawa M, Mori E, Kuwana N; Study of INPH on neurological improvement (SINPHONI). Diagnosis of idiopathic normal pressure hydrocephalus is supported by MRI-based scheme: a prospective cohort study. Cerebrospinal Fluid Res. 2010 Oct 31;7:18. doi: 10.1186/1743-8454-7-18. PMID: 21040519; PMCID: PMC2987762. 